\title{
Abnormal Aortic Wall Properties in Women with Turner Syndrome
}

\author{
Lidia R. Bons, MD, PhD ${ }^{1,2}$ Allard T. Van Den Hoven, MD ${ }^{10} \quad$ Maira Malik, $\mathrm{BSc}^{1}$
}

Annemien E. Van Den Bosch, MD, PhD ${ }^{1}$ Jacky S. McGhie, $\mathrm{PhD}^{1}$ Anthonie L. Duijnhouwer, MD ${ }^{30}$ Hans-Marc J. Siebelink, MD, PhD Alexander Hirsch, MD, PhD ${ }^{1,2}$ Daniel H. Devos, MD, PhD Ernst Rietzschel, MD, $\mathrm{PhD}^{6}$ Jan H. von der Thüsen, MD, PhD ${ }^{7}$ Ingrid M.B.H. van de Laar, MD, $\mathrm{PhD}^{8}$ Judith M.A. Verhagen, MD, $\mathrm{PhD}^{8}$ Ingrid van der Pluijm, $\mathrm{PhD}^{9,10}$ Ricardo P.J. Budde, MD, $\mathrm{PhD}^{1,2}$ Jolien W. Roos-Hesselink, MD, $\mathrm{PhD}^{1}$ (1)

\footnotetext{
${ }^{1}$ Department of Cardiology, Erasmus MC, University Medical Center Rotterdam, Rotterdam, The Netherlands

2 Department of Radiology and Nuclear Medicine, Erasmus MC, University Medical Center Rotterdam, Rotterdam, The Netherlands

${ }^{3}$ Department of Cardiology, Radboud University Medical Center, Nijmegen, The Netherlands

${ }^{4}$ Department of Cardiology, Leiden University Medical Center, Leiden, The Netherlands

${ }^{5}$ Department of Radiology, Ghent University Hospital, Gent, Belgium

${ }^{6}$ Department of Cardiology, Ghent University Hospital, Gent, Belgium

7 Department of Pathology, Erasmus MC, University Medical Center Rotterdam, Rotterdam, The Netherlands

8 Department of Clinical Genetics, Erasmus MC, University Medical Center Rotterdam, Rotterdam, The Netherlands

${ }^{9}$ Department of Vascular Surgery, Erasmus MC, University Medical Center Rotterdam, Rotterdam, The Netherlands

10 Department of Molecular Genetics, Erasmus MC, University Medical Center Rotterdam, Rotterdam, The Netherlands
}

\author{
Address for correspondence Jolien W. Roos-Hesselink, MD, PhD, \\ Department of Cardiology, Erasmus MC, Room Rg-435, 's \\ Gravendijkwal 230, 3015 CE Rotterdam, The Netherlands \\ (e-mail: j.roos@erasmusmc.nl).
} AORTA 2020;8:121-131.
Abstract
Keywords
- Turner syndrome
- aortic stiffness
- pulse wave velocity
- echocardiography
- cardiovascular magnetic resonance

Background Turner syndrome (TS) is associated with aortic dilatation and dissection, but the underlying process is unclear. The aim of this study was to investigate the elastic properties and composition of the aortic wall in women with TS.

Methods In this cross-sectional study, 52 women with TS aged $35 \pm 13$ years (50\% monosomy, 12 with bicuspid aortic valve [BAV] and 4 with coarctation) were investigated using carotid-femoral pulse wave velocity (CF-PWV) by echocardiography and ascending aortic distensibility (AAD) and aortic arch pulse wave velocity (AA-PWV) by magnetic resonance imaging (MRI). As control group, 13 women with BAV without TS and 48 healthy patients were included.

Results Women with TS showed a higher AA-PWV $(\beta=1.08$, confidence interval [CI]: $0.54-1.62)$ after correcting for age and comorbidities compared with controls. We found no significant difference in AAD and CF-PWV. In women with TS, the presence of BAV, coarctation of the aorta, or monosomy $(45, \mathrm{X})$ was not associated with aortic stiffness. In addition, aortic tissue samples were investigated with routine and immunohistochemical stains in five additional women with TS who were operated. received

November 8, 2019 accepted after revision May 30, 2020
DOI https://doi.org/

10.1055/s-0040-1714384. ISSN 2325-4637.
Copyright $\odot 2020$ by Thieme Medical Publishers, Inc., 333 Seventh Avenue, New York, NY 10001, USA. Tel: +1(212) 760-0888.
License terms

(c) (i) 
The tissue showed more compact smooth muscle cell layers with abnormal deposition and structure of elastin and diminished or absent expression of contractile proteins desmin, actin, and caldesmon, as well as the progesterone receptor.

Conclusion Both aortic arch stiffness measurements on MRI and histomorphological changes point toward an inherent abnormal thoracic aortic wall in women with TS.

\section{Introduction}

Turner syndrome (TS) is a genetic condition caused by partial or complete absence of an X chromosome and occurs in 50 per 100,000 females at birth. ${ }^{1}$ The main complication is aortic dissection, most often preceded by aortic dilatation, ${ }^{2}$ which carries a substantial mortality risk. ${ }^{3}$ To prevent acute aortic dissection, preventive surgery is advised when the aortic diameter exceeds a diagnosis-specific cut-off value. Since patients with TS are known for their short stature, correction for body size area using the aortic size index is advised. ${ }^{4}$ However, even patients with a normal aortic size index may develop aortic dissection. ${ }^{5}$ Consequently, aortic dilatation alone is not sufficient for identifying patients with TS at high risk of aortic dissection. Other clinical parameters identifying high-risk patients are not very clear. ${ }^{6}$

It has been proposed that other more subtle changes such as increased stiffness of the aortic wall, are present in patients with TS prior to dissection. Studies in patients with hypertension, diabetes, or ischemic heart disease have shown aortic stiffness to be an independent predictor of all-cause mortality and cardiovascular events. ${ }^{7}$ Although previous literature showed an increased aortic stiffness in patients with $\mathrm{TS}^{8-10}$ it remains unclear to what extent this is explained by the commonly found bicuspid aortic valve (BAV) and coarctation of the aorta (CoA). Both BAV and CoA are also associated with aortic stiffness, ${ }^{11,12}$ and it is known that dissection may occur in the absence of BAV and CoA. ${ }^{13}$ Differences in aortic stiffness between monosomy $(45, X)$ and mosaicism have also not yet been investigated.

In addition, histomorphological examination of aortic tissue from patients might also be of help to identify possible pathological changes in the aortic wall and to interpret aortic stiffness imaging parameters.

The aim of this current study was to investigate whether the aortic wall of patients with TS is different from that of healthy control patients, and if this can be explained solely by the bicuspid valve and/or aortic coarctation.

\section{Materials and Methods}

In this cross-sectional cohort study, we included all adult patients with TS who participated between October 2014 and March 2016 in a study previously described. ${ }^{14}$ Inclusion criteria for patients with TS were a $45, \mathrm{X}$ or $45, \mathrm{X} / 46$, and XX mosaic karyotype. The patients were prospectively included and subjected to physical examination (including height, weight, and systolic and diastolic blood pressure), electrocardiography, two- and three-dimensional (2D and 3D, respec- tively) echocardiography, computed tomography angiography (CTA), and magnetic resonance imaging (MRI), all on the same day.

Measurements of the aortic diameter were performed using the double-oblique technique on CTA during systole, except for two patients. In those two patients, contrastenhanced magnetic resonance angiography (CEMRA) images were used, as CTA was not performed. Scan parameters of the CT imaging can be found elsewhere. ${ }^{14}$

Two reference groups were selected: patients with BAV without TS and healthy control women. We selected patients with BAV from the same prospective cohort study from which the patients with TS were included. The inclusion criteria for BAV were age $\geq 18$ years and one of the following features: (1) aortic stenosis (gradient $>2.5 \mathrm{~m} / \mathrm{sec}$ ), (2) aortic regurgitation (at least moderate), or (3) ascending aortic diameter $\geq 40 \mathrm{~mm}$ and/or aortic size index $>2.1$.

For carotid-femoral pulse wave velocity (CF-PWV) measurements, we included a statistically age-matched control group. They did not undergo CTA or MRI and therefore aortic diameters were measured during midsystole with echocardiography in this group of healthy patients using the leadingedge-to-leading-edge technique.

The healthy control group for aortic distensibility and aortic arch pulse wave velocity (AA-PWV) measurements were recruited from a previous MRI study on aortic stiffness of patients with the same age. ${ }^{15}$

The study was approved by the medical ethical committee (MEC14-225). Written informed consent was provided by all patients and controls.

A standard 2D transthoracic echocardiogram was performed by an experienced sonographer. All studies were acquired using harmonic imaging on an iE33 or EPIQ7 ultrasound system (Philips Medical Systems, Best, the Netherlands) equipped with an x5-1 matrix-array transducer (composed of 3040 elements operating at $1-5 \mathrm{MHz}$ ). The aorta was measured in the standard parasternal long axis view or on a more cranial view one intercostal space higher which often improved the visualization of the ascending aorta.

PW Doppler signals were obtained consecutively at the common carotid artery and the femoral artery (-Supplementary Fig. S1, panel A). PWV (expressed in $\mathrm{m} / \mathrm{s}$ ) was defined as: distance (in meters) between carotid artery and femoral artery/delta time (in seconds). The distance was measured outside the patient's body with a measuring tape. For both signals, the time interval was measured between the onset of the QRS complex and the onset of the systolic PW Doppler signal. Delta time was 
calculated as the difference between both time intervals. Validated software was used to analyze the PWV (Curad Viewer version 3.5.3.0, Wijk bij Duurstede, the Netherlands).

Image acquisition was performed using a 1.5-T scanner (Discovery MR450, GE Medical Systems, Milwaukee, WI, USA) using a 32-channel phased-array cardiac surface coil. A retrospectively echocardiography (ECG)-gated 2D balanced steadystate free precession sequence during breath hold was obtained perpendicular to the ascending aorta at the level of the pulmonary trunk to measure aortic distensibility. Typical scan parameters were as follows: field of view (FOV) $420 \mathrm{~mm}$ (phase FOV 60\%); matrix size, $256 \times 192$; slice thickness, $6.5 \mathrm{~mm}$; flip angle, 45 degrees; repetition time/echo time (TR/TE), 3.3/1.5 ms; and number of reconstructed phases, 24 per cardiac cycle. The contour of the ascending aorta was manually traced in all phases (QMass analytical software version 8.1, Medis, Leiden, the Netherlands). The minimum and maximum ascending aortic cross-sectional areas were identified, and aortic distensibility (10-3 $\mathrm{mm} \mathrm{Hg}^{-1}$ ) was calculated using the following calculation: (maximum area minimum area $) /($ minimum area $\times \Delta \mathrm{P}) \times 1,000$, where $\Delta \mathrm{P}$ is the brachial pulse pressure in $\mathrm{mmHg}$ (-Supplementary Fig. S1, panel B).

A free-breathing, retrospectively ECG-gated 2D phase-contrast flow sequence was performed to measure aortic PWV. The slice plane was positioned at the level of the pulmonary artery perpendicular to the ascending and descending aorta. Typical scan parameters were as follows: FOV, $350 \mathrm{~mm}$ (phase FOV $90 \%$ ); matrix size, $192 \times 160$; slice thickness, $7.0 \mathrm{~mm}$; flip angle, 20 degrees; number of averages, 3; TR/TE, 4.3/2.3 ms; views per segment, 1; velocity encoding value, $200 \mathrm{~cm} / \mathrm{s}$; true temporal resolution, approximately $11 \mathrm{~ms}$; and number of reconstructed phases, 100 per cardiac cycle. AA-PWV was defined as distance between ascending and descending aorta/ $\Delta$ time (expressed in $\mathrm{m} / \mathrm{s}$ ). Delta time was measured with the same method explained by Devos et $\mathrm{al}^{9}$ (-Supplementary Fig. S1, panel C). The onset of the systolic wave front was determined from the resulting flow graph by the intersection point of the constant horizontal diastolic flow and the upslope of the systolic wave front, the latter of which was modeled by linear regression along the upslope from the flow values between 20 and $80 \%$ of the total range. To determine the length of the aortic segment between the two aortic levels, sagittal angulated T1-weighted black blood turbo spin echo images of the AA were acquired during breath-hold. Typical scan parameters were FOV, $400 \mathrm{~mm}$ (phase FOV 100\%); matrix, $256 \times 256$; slice thickness, $5.0 \mathrm{~mm}$; and flip angle, 155 degrees. When the AA was not properly visible on the black blood images a multiplanar reconstruction was made with CEMRA images to measure the distance $(n=35,47 \%)$. The CEMRA scan parameters are described elsewhere. ${ }^{14}$ Qmass software was used for distensibility measurements and Qflow software was used for PWV measurements (Medis, Leiden, the Netherlands).

We studied the histomorphology of formalin-fixed paraffin-embedded (FFPE) specimens of the ascending aorta of five TS patients who underwent preventive replacement of the ascending aorta because of dilatation. In one patient, we additionally performed electron microscopy examination to ultrastructurally investigate medial elastin fiber morphology and deposition of elastin. To clearly show the differences between aortic tissue of patients with TS and healthy tissue, aortic tissue from a healthy woman aged 45 years was obtained from the Heart Valve Bank in Rotterdam and investigated.

Routine staining with hematoxylin-eosin (HE), the elastica van Gieson (EvG), and alcian blue (AB) stains was performed on $3 \mu \mathrm{m}$ sections. The criteria as outlined in the recent consensus statement on surgical pathology of the aorta ${ }^{16}$ were used for scoring and classification of abnormalities. In addition, immunohistochemical stains were performed on a Ventana Benchmark Ultra-automated staining platform (Roche Diagnostics, Tucson, AZ) to assess the vessel wall expression of smooth muscle actin (SMA), caldesmon, desmin, actin HHF-35, estrogen receptor (ER), and progesterone receptor (PR), as well as the proliferation marker MIB-1. Immunohistochemical methods including the antibody clones used are provided in the Supplementary Material (- Supplementary Appendix A). For electron microscopy, representative samples of the media were isolated from paraffin blocks and incubated with a mixture of $1 \%$ glutaraldehyde and $4 \%$ formaldehyde. The sections were osmicated with $1 \%$ osmium in distilled water, dehydrated in 50 to $100 \%$ Acetone and embedded in a mixture of Embed-812, NMA, DDSA, and DMP-30 (EMS diasum, Hatfield, Pennsylvania). Ultrathin sections (60-70 nm) were cut using an Ultramicrotome UC7 (Leica, Germany), mounted on copper grids, and counterstained with uranyl acetate $0,5 \%$ for 30 minutes at $45^{\circ} \mathrm{C}$ and Ultrostain II (Leica, Germany) for 7 minutes at room temperature in the Leica AC20. Elastin lamellae were visualized at magnifications of $\times 2,800$ and $\times 7,100$ and analyzed under the electron microscope (Philips, Eindhoven, the Netherlands).

All characteristics are presented as mean \pm standard deviation when normally distributed, and in case of nonnormal distribution, medians (interquartile ranges [IQR]) are provided. Categorical variables are presented as frequencies with percentages. Comparison of normally distributed continuous variables between two groups was done using the Student's t-test or, in case of a skewed distribution, the Mann-Whitney test. Univariable linear regression analysis was performed with patient characteristics as independent variables and the measurement of aortic stiffness as dependent variable. In addition, multivariable analysis was performed using the Enter method to adjust for age, aortic size index, BAV, CoA, and hypertension. To assess interobserver variability, the intraclass correlation coefficient (ICC) was calculated for all measurements using 25 randomly selected patients. The IBM SPSS statistics 21.0 software was used to analyze the data. The statistical tests were two sided and a $p$ value below 0.05 was considered significant.

\section{Results}

In total, 52 women with TS aged $35 \pm 14$ years were included: 40 with a tricuspid aortic valve (TAV) and 12 patients with BAV. Twenty-five TS women had X chromosome monosomy $(45, \mathrm{X})$ and the other 25 TS women had X chromosome 
mosaicism ( $n=2$ missing). Renal function was normal in all. In 13 women (25\%), the time between the first and last investigation was $\geq 1$ day (median, 7 days; IQR: $5-42$ days) due to technical or practical issues. We included 13 women with BAV without TS and 10 healthy women for the control groups of CF-PWV. The control group for aortic distensibility and AA-PWV measurements consisted of the same 13 women with BAV and an additional 38 healthy female patients. In - Fig. 1, a flowchart is presented with the number of measurements that could be performed in the participants.
- Table 1 shows the baseline patient characteristics of the TS women and control groups separately. Surgical correction for CoA with end-to-end anastomosis was performed in three patients with TS. Five patients with TS were treated for hypertension with $\beta$-blockers, angiotensin receptor blockers, or angiotensin converting enzyme (ACE) inhibitors.

-Figure 2 shows the comparison between patients with TS and controls for the CF-PWV, ascending aortic distensibility and AA-PWV. We found no significant difference in CFPWV between the groups. Within the women with TS,

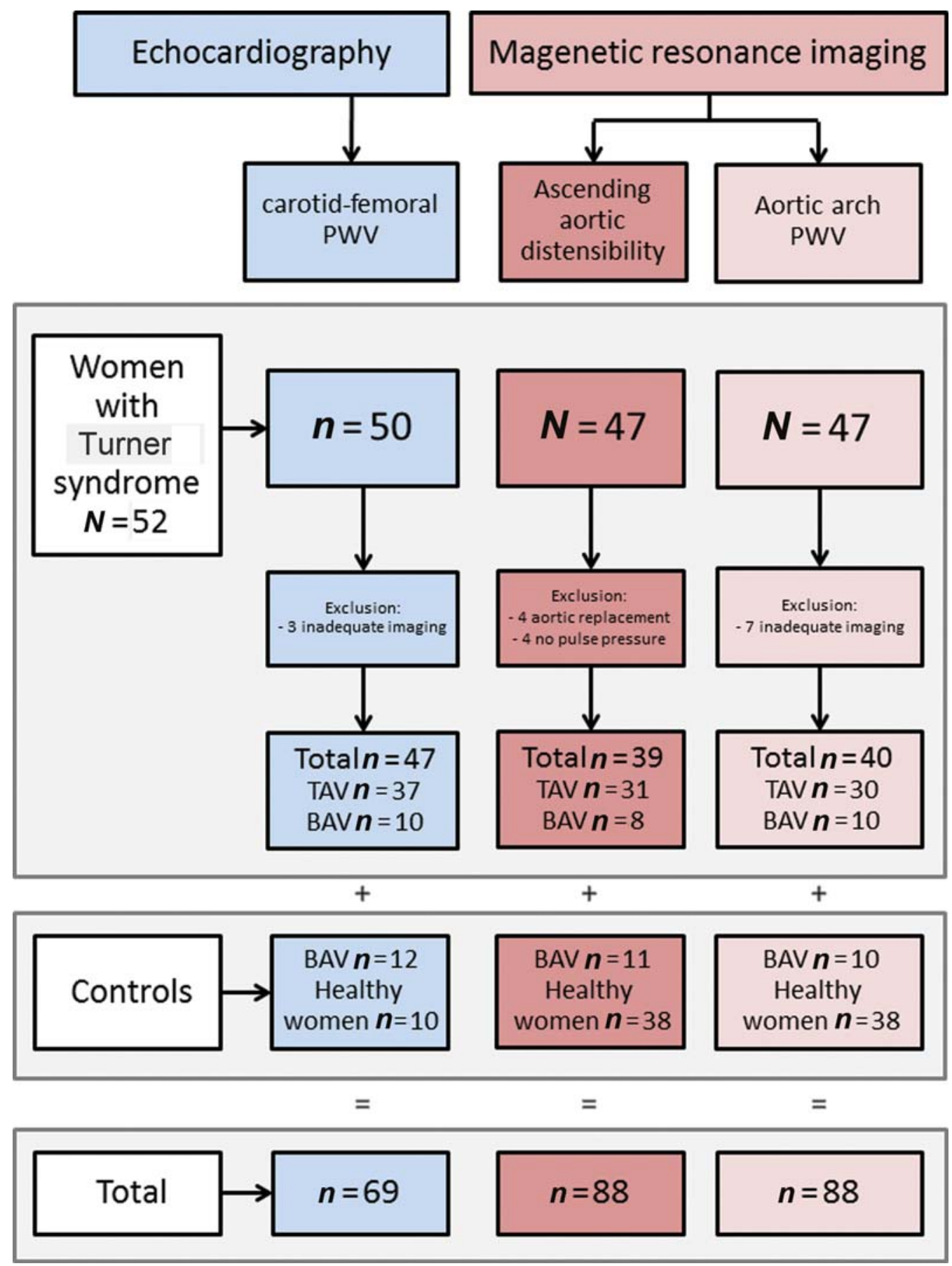

Fig. 1 Flow chart of the patients with aortic stiffness measurements. All three measurements could be performed in 32 patients with Turner syndrome (25 TAV and $7 \mathrm{BAV}$ ) and in 8 control patients with BAV only. BAV, bicuspid aortic valve; PWV, pulse wave velocity; TAV, tricuspid aortic valve. 
Table 1 Baseline characteristics

\begin{tabular}{|c|c|c|c|c|c|c|}
\hline \multirow[t]{2}{*}{ Characteristics } & \multicolumn{3}{|c|}{ Turner syndrome $(n=52)$} & \multirow{2}{*}{$\begin{array}{l}\text { Bicuspid aortic valve } \\
\text { without Turner } \\
\text { syndrome }(n=13)\end{array}$} & \multirow{2}{*}{$\begin{array}{l}\text { Healthy controls } \\
\text { echocardiography } \\
(n=10)\end{array}$} & \multirow{2}{*}{$\begin{array}{l}\text { Healthy } \\
\text { controls MRI } \\
(n=38)\end{array}$} \\
\hline & $\begin{array}{l}\text { Total } \\
(n=52)\end{array}$ & $\begin{array}{l}\text { TAV } \\
(n=40)\end{array}$ & $\begin{array}{l}\text { BAV } \\
(n=12)\end{array}$ & & & \\
\hline Age (y) & $35 \pm 14$ & $36 \pm 14$ & $35 \pm 13$ & $34 \pm 11$ & $35 \pm 15$ & $34 \pm 14$ \\
\hline Height (cm) & $156 \pm 9$ & $157 \pm 9$ & $154 \pm 7$ & $169 \pm 11$ & $187 \pm 6$ & $166 \pm 8$ \\
\hline Weight $(\mathrm{kg})$ & $66 \pm 16$ & $68 \pm 17$ & $61 \pm 11$ & $69 \pm 11$ & $83 \pm 10$ & $61 \pm 11$ \\
\hline $\begin{array}{l}\text { Systolic blood } \\
\text { pressure }(\mathrm{mm} \mathrm{Hg})\end{array}$ & $124 \pm 17$ & $124 \pm 18$ & $126 \pm 16$ & $116 \pm 12$ & $131 \pm 10$ & $118 \pm 14$ \\
\hline $\begin{array}{l}\text { Diastolic blood } \\
\text { pressure }(\mathrm{mm} \mathrm{Hg})\end{array}$ & $80 \pm 13$ & $80 \pm 12$ & $83 \pm 14$ & $77 \pm 7$ & $79 \pm 7$ & $70 \pm 9$ \\
\hline Hypertension & $8(15 \%)$ & $7(18 \%)$ & $1(8 \%)$ & $3(23 \%)$ & $0(0 \%)$ & $0(0 \%)$ \\
\hline $\begin{array}{l}\text { Surgical correction } \\
\text { for aortic coarctation }^{\text {a }}\end{array}$ & $3(6 \%)$ & $0(0 \%)$ & $3(25 \%)$ & $2(15 \%)$ & $0(0 \%)$ & $0(0 \%)$ \\
\hline $\begin{array}{l}\text { Aortic dilatation } \\
\left(>40 \mathrm{~mm} \text { and } / \mathrm{or}^{2}\right. \\
\left.\text { ASI }>2.1 \mathrm{~cm} / \mathrm{cm}^{2}\right)\end{array}$ & $11(21 \%)$ & $6(15 \%)$ & $5(42 \%)$ & $9(69 \%)$ & $0(0 \%)$ & $1(3 \%)^{b}$ \\
\hline $\begin{array}{l}\text { Aortic stenosis } \\
\left(\text { echo } V_{\max }>2.5 \mathrm{~m} / \mathrm{s}\right)\end{array}$ & $1(2 \%)$ & $0(0 \%)$ & $1(8 \%)$ & $11(85 \%)$ & $0(0 \%)$ & $0(0 \%)$ \\
\hline $\begin{array}{l}\text { Aortic regurgitation } \\
\text { (moderate or severe) }\end{array}$ & $1(2 \%)$ & $0(0 \%)$ & $1(8 \%)$ & $5(39 \%)$ & $0(0 \%)$ & $0(0 \%)$ \\
\hline $\begin{array}{l}\text { Absolute ascending } \\
\text { aortic diameter ( } \mathrm{mm})\end{array}$ & $31 \pm 5$ & $30 \pm 4$ & $33 \pm 6$ & $40 \pm 7$ & $31 \pm 3$ & $27 \pm 3$ \\
\hline $\begin{array}{l}\text { Aortic size index } \\
\text { ascending aorta }\left(\mathrm{mm} / \mathrm{m}^{2}\right)\end{array}$ & $19 \pm 4$ & $18 \pm 3$ & $21 \pm 3$ & $22 \pm 3$ & $15 \pm 2$ & $16 \pm 2$ \\
\hline
\end{tabular}

ASI, aortic size index (aortic diameter divided by body surface area); BAV, bicuspid aortic valve; MRI, magnetic resonance imaging; TAV, tricuspid aortic valve; $\mathrm{V}_{\max }$, maximum velocity.

Note: data are expressed as mean \pm standard deviation or $n$ (percentage).

Missing values were present for height $(n=1)$ and weight $(n=1)$, blood pressure $(n=6)$ and aortic size index $(n=1)$.

${ }^{a}$ One additional Turner patient has a coarctation of the aorta, but he did not undergo surgical correction.

${ }^{\mathrm{b}}$ One healthy woman with an aortic diameter of $29 \mathrm{~mm}$, height of $151 \mathrm{~cm}$ and weight of $40 \mathrm{~kg}$, which resulted in an ASI of $2.2 \mathrm{~cm} / \mathrm{m}^{2}$.

\section{Carotid-femoral PWV}

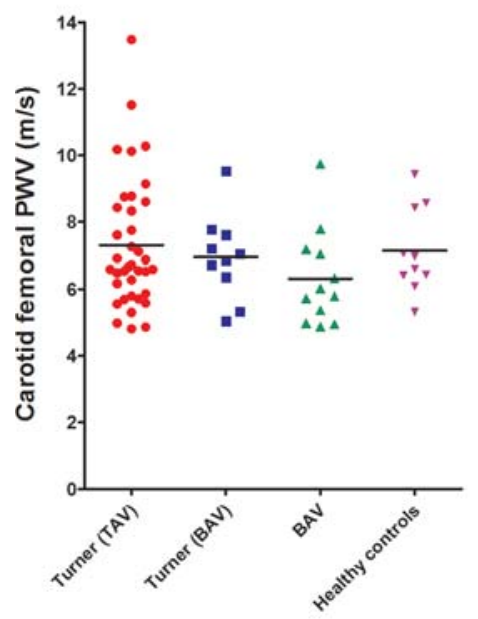

Ascending aortic distensibility

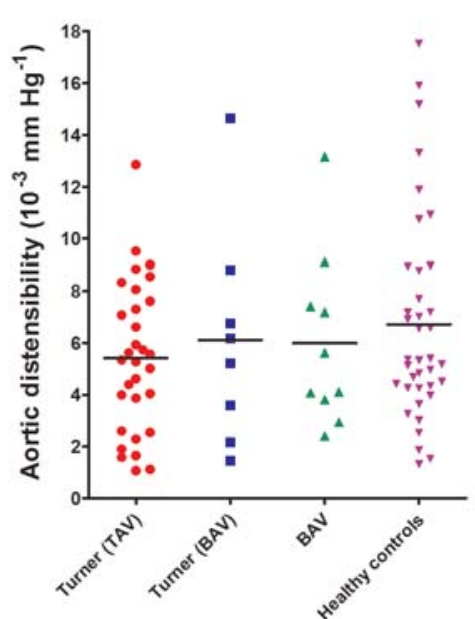

Aortic arch PWV

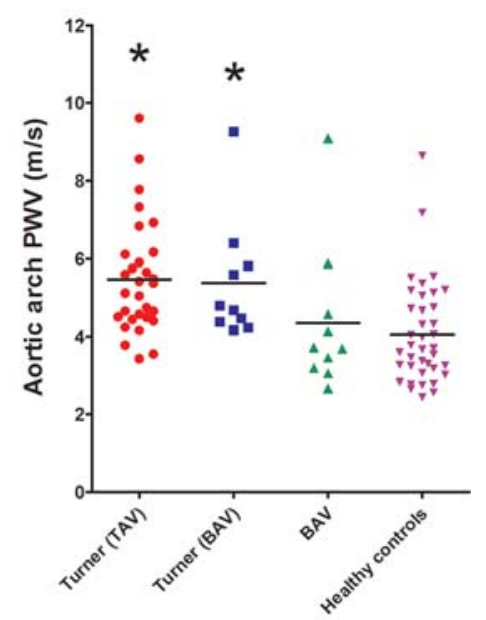

Fig. 2 Uncorrected results of carotid-femoral pulse wave velocity, ascending aortic distensibility, and aortic arch pulse wave velocity divided into four groups. The median level in each subgroup is indicated with the black line. BAV, bicuspid aortic valve; PWV, pulse wave velocity; TAV, tricuspid aortic valve. * Significantly different from the healthy group according to the Wilcoxon's one-sample test. 
patients with BAV (median = 7.0, IQR: $6.1-7.7 \mathrm{~m} / \mathrm{s}$ ) did not show a significantly different CF-PWV compared with the patients without BAV (median =6.7, IQR: $5.8-8.5 \mathrm{~m} / \mathrm{s}$ ). In all four TS women with CoA, the CF-PWV could be measured $(5.3,6.9,7.6$, and $7.8 \mathrm{~m} / \mathrm{s})$ and was not statistically different from TS women without CoA (median $=6.7$, IQR: $5.9-8.4$ $\mathrm{m} / \mathrm{s}$ ). Also, no statistical difference was found between patients with monosomy (median $=6.5$, IQR: $5.7-7.7 \mathrm{~m} / \mathrm{s}$ ) or mosaicism (median =6.9, IQR: 6.4-8.6 m/s). For CF-PWV measurements we found an ICC of 0.893 (0.772-0.951).

We found no significant difference in ascending aortic distensibility between the groups. Within the women with TS, patients with BAV (median = 5.7, IQR: $2.5-8.3 \mathrm{~mm} \mathrm{Hg}^{-1}$ ) did not show a significantly different ascending aortic distensibility compared with the patients without $\mathrm{BAV}$ ( median $=5.3$, IQR: $2.6-7.6 \mathrm{~mm} \mathrm{Hg}^{-1}$ ). Only in two TS women with CoA, the ascending aortic distensibility could be measured (1.5 and $6.2 \times 10^{-3} \mathrm{~mm} \mathrm{Hg}^{-1}$ ). No statistical difference was found between patients with monosomy (median $=5.2, \mathrm{IQR}$ : 3.9-7.1 mm $\mathrm{Hg}^{-1}$ ) or mosaicism (median=5.6, IQR: 2.3-8.3 $\mathrm{mm} \mathrm{Hg}^{-1}$ ). For ascending aortic distensibility measurements we found an ICC of 0.999 (0.996-0.999) for the minimum area and 0.999 (0.998-1.000) for the maximum area.

Patients with TS showed significantly higher AA-PWV compared with healthy controls (-Fig. 2). In the multivariable analysis ( $\mathbf{- T a b l e ~} \mathbf{2}$ ), the presence of TS remained signif- icantly associated with higher AA-PWV $(\beta=1.08$, 95\% confidence interval $[\mathrm{CI}]: 0.54-1.62, p<0.001)$. Within the patients with TS, patients with BAV (median $=4.8$, IQR: 4.4-6.0 m/s) did not show a significantly different AAPWV compared with the patients without BAV (median = 5.3, IQR: 4.5-6.1 m/s). In three TS women with CoA, the AA-PWV could be measured (4.4, 5.8, and $6.4 \mathrm{~m} / \mathrm{s})$, which was comparable to the median of TS women without CoA (median =4.9, IQR: $4.5-5.9 \mathrm{~m} / \mathrm{s}$ ). No statistical difference was found between patients with monosomy (median $=4.7$, IQR: $4.4-5.8 \mathrm{~m} / \mathrm{s}$ ) or mosaicism (median = 5.5, IQR: 4.5-6.2 $\mathrm{m} / \mathrm{s}$ ). For AA-PWV measurements, we found an ICC of 0.970 (0.935-0.987) for the distance measurements and 0.704 (0.437-0.857) for the time measurements.

The five patients with TS who underwent surgery had an age range of 11 to 47 years at time of surgery. - Supplementary Table S1 shows a complete overview of the routine stains of each patient. Using the criteria outlined in the recent consensus statement, the patients were found to show only mild medial degeneration in four patients, and no medial degeneration in one patient, with mostly intralamellar and focal, mucoid matrix accumulation (-Fig. 3). In addition to these findings, we found compact smooth muscle cell layers and a decrease in the intralamellar space, but only mild smooth muscle cell nuclei loss. Also, conspicuous granular deposition of elastin adjacent to lamellae was found

Table 2 Univariable and multivariable analysis of aortic stiffness measurements in the total group including patients with Turner syndrome, bicuspid aortic valve patients and control patients

\begin{tabular}{|c|c|c|c|c|c|}
\hline \multicolumn{2}{|l|}{ Characteristics } & \multicolumn{2}{|l|}{ Univariable analysis } & \multicolumn{2}{|l|}{ Multivariable analysis $^{a}$} \\
\hline & & Beta $(95 \% \mathrm{Cl})$ & $p$-Value & Beta $(95 \% \mathrm{Cl})$ & $p$-Value \\
\hline \multirow{6}{*}{$\begin{array}{l}\text { Carotid-femoral } \\
\text { PWV }(n=69)\end{array}$} & Turner Syndrome & $0.54(-0.34 ; 1.42)$ & 0.223 & $0.03(-0.65 ; 0.72)$ & 0.924 \\
\hline & Age & $0.09(0.06 ; 0.11)$ & $<0.001$ & $0.06(0.04 ; 0.09)$ & $<0.001$ \\
\hline & Baseline aortic size index & $0.16(0.05 ; 0.27)$ & 0.004 & $0.14(0.04 ; 0.25)$ & 0.007 \\
\hline & Bicuspid aortic valve & $-0.67(-1.54 ; 0.21)$ & 0.132 & $-1.03(-1.95 ;-0.12)$ & 0.028 \\
\hline & Aortic coarctation & $-0.42(-2.01 ; 1.16)$ & 0.596 & $0.53(-0.76 ; 1.82)$ & 0.413 \\
\hline & Hypertension & $2.41(1.26 ; 3.56)$ & $<0.001$ & $1.082(0.13 ; 2.03)$ & 0.027 \\
\hline \multirow{6}{*}{$\begin{array}{l}\text { Ascending aortic } \\
\text { distensibility } \\
\left(n=87^{\mathrm{b}}\right)\end{array}$} & Turner Syndrome & $-1.01(-2.5 ; 0.51)$ & 0.191 & $-0.87(-1.99 ; 0.25)$ & 0.126 \\
\hline & Age & $-0.18(-0.22 ;-0.14)$ & $<0.001$ & $-0.18(-0.23 ;-0.14)$ & $<0.001$ \\
\hline & Baseline aortic sixe index & $-0.15(-0.36 ; 0.07)$ & 0.181 & $0.11(-0.11 ; 0.32)$ & 0.327 \\
\hline & Bicuspid aortic valve & $-0.09(-1.97 ; 1.79)$ & 0.926 & $-0.33(-2.17 ; 1.52)$ & 0.726 \\
\hline & Aortic coarctation & $-1.38(-5.00 ; 2.24)$ & 0.451 & $-1.86(-4.79 ; 1.07)$ & 0.210 \\
\hline & Hypertension & $-3.61(-6.13 ;-1.09)$ & 0.005 & $-1.25(-3.35 ; 0.85)$ & 0.238 \\
\hline \multirow{6}{*}{$\begin{array}{l}\text { Aortic arch } \\
\text { PWV }\left(n=87^{b}\right)\end{array}$} & Turner Syndrome & $1.34(0.72 ; 1.95)$ & $<0.001$ & $1.08(0.54 ; 1.62)$ & $<0.001$ \\
\hline & Age & $0.06(0.04 ; 0.08)$ & $<0.001$ & $0.05(0.03 ; 0.07)$ & $<0.001$ \\
\hline & Baseline aortic sixe index & $0.14(0.05 ; 0.24)$ & 0.004 & $0.06(-0.03 ; 0.16)$ & 0.198 \\
\hline & Bicuspid aortic valve & $0.18(-0.62 ; 0.99)$ & 0.651 & $-0.35(-1.17 ; 0.47)$ & 0.395 \\
\hline & Aortic coarctation & $1.39(0.27 ; 2.50)$ & 0.015 & $0.66(-0.35 ; 1.67)$ & 0.195 \\
\hline & Hypertension & $2.47(1.42 ; 3.52)$ & $<0.001$ & $0.80(-0.24 ; 1.85)$ & 0.131 \\
\hline
\end{tabular}

Abbreviations: PWV, pulse wave velocity.

Note: bold = statistical significant in multivariabele analysis.

${ }^{a}$ Corrected for all other variables mentioned in this table.

bone patient had a missing value for weight. 

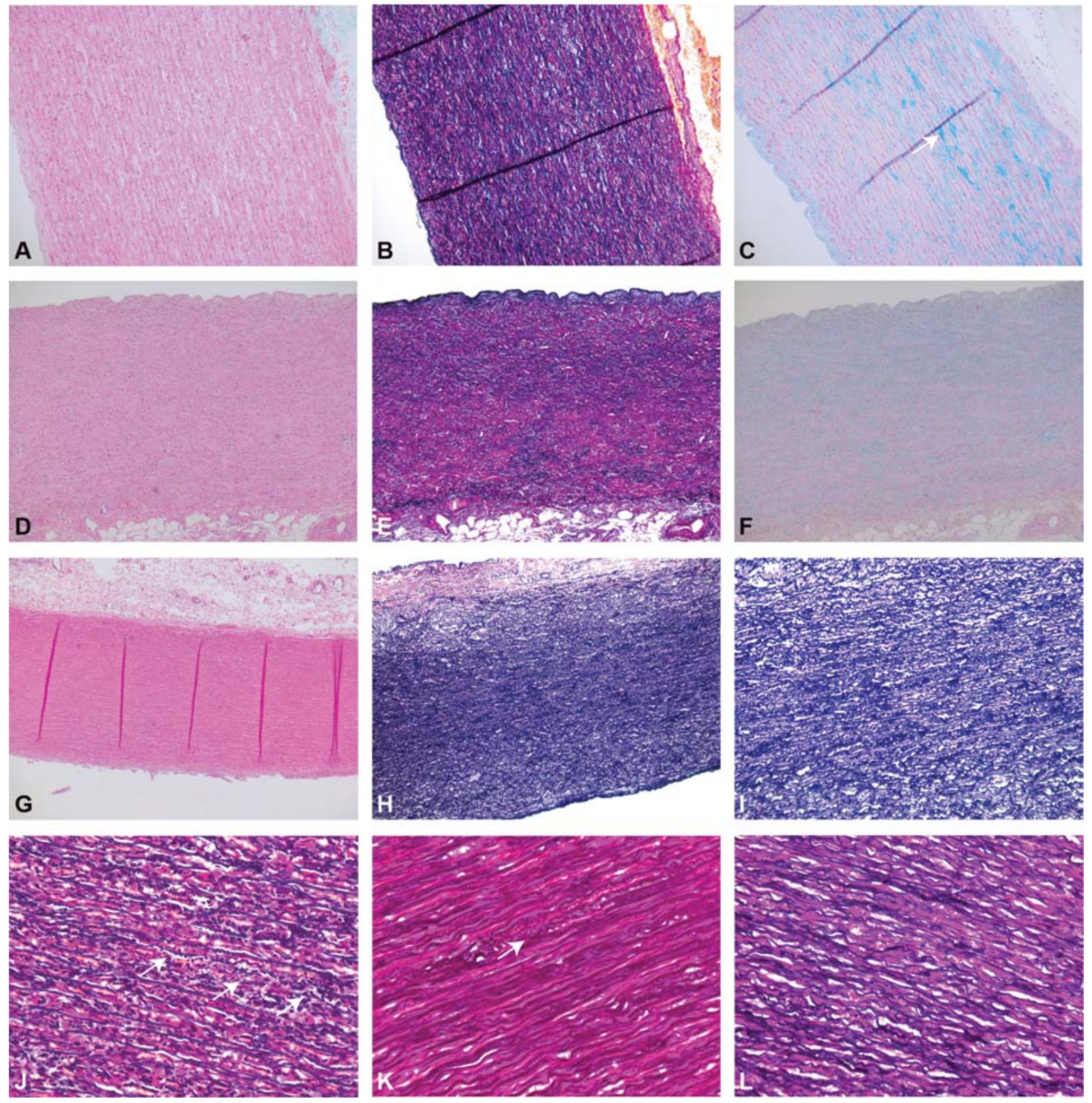

Fig. 3 Routine stains of aortic tissue of patients with Turner syndrome and healthy controls. Top row: (A) hematoxylin-eosin, (B) Elastica-van Gieson, and (C) alcian blue stains of a representative aortic explant of a Turner syndrome patient, with essentially intact lamellation, and only minimal elastin fragmentation (B) and mucin deposition (C, arrow). Second row: (D) control case lacking significant degeneration in hematoxylin-eosin stains, (E) Elastica-van Gieson stains, or (F) alcian blue stains. Third row: (G) Turner case lacking significant degeneration in hematoxylin-eosin stains or (H, I) Elastica-van Gieson stains, but with decrease in smooth muscle cell volume. Bottom row: (J) Elastica-van Gieson stains of two Turner patients with extensive and (K) focal granular deposition of elastin fibers (arrows) and (L) one healthy control (L).

in aortic tissue of women with TS when compared with healthy controls (-Fig. 3). Additional electron microscopic images confirmed these granular structures by showing "bulges" on the surface of elastin fibers (-Fig. 4). The elastin fibers themselves were positioned closer together and were more irregular and thinner compared with a healthy control. Immunohistochemically, the most striking findings were seen in the middle section of the media with a complete lack of desmin expression in all five patients, underexpression of caldesmon and progesterone receptor in four out of five, and weak expression of HHF-35 in three out of five patients (-Fig. 5). We observed apparent overexpression of estrogen receptor in the media of TS patients, while SMA and MIB-1 were not differentially expressed.

\section{Discussion}

The results of our study show that women with TS have a stiffer AA compared with controls, independent of the presence of BAV or CoA. Ascending aortic distensibility and CF-PWV were not statistically different between patients with TS and controls. We also found no effect of karyotype on 

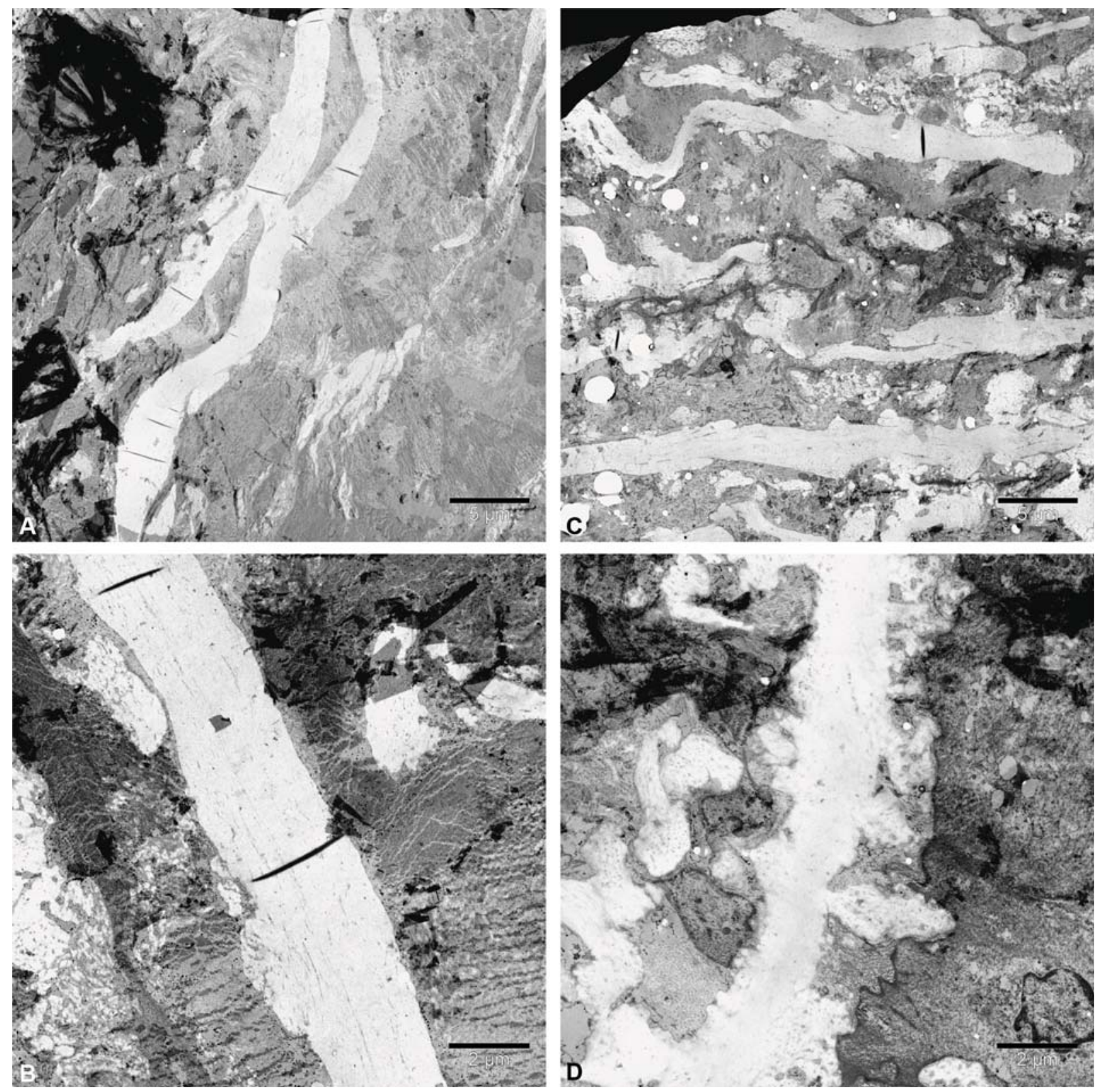

Fig. 4 Representative electron microscopic images of the aortic media of a women with Turner syndrome (A and B) and a healthy control (C and D). $(A$ and $C) \times 2,800$ magnification, $(B$ and $D) \times 7,100$ magnification. This figure shows that the elastin lamellae of the women with Turner syndrome are positioned closer together, more irregular and thinner, and display numerous excrescences on the surface.

aortic stiffness. Our imaging results confirm that the wall of the aorta is indeed different in patients with TS, even in the absence of cardiovascular pathology, such as a bicuspid valve, and this seems especially true for the AA and ascending aorta. In addition, clear histomorphological changes are found in the ascending aorta, pointing toward an inherent abnormal aortic wall.

The observed increased AA-PWV in our study was also observed by Devos et al ${ }^{9}$; however, Schäfer et $\mathrm{al}^{8}$ did not find these results in the younger group of patients with TS. This may indicate that aortic stiffness occurs later in the disease process. Besides increased aortic stiffness, patients with TS also have a higher risk of AA abnormalities, such as elongation of the AA. ${ }^{17}$ It might be that anatomical abnormalities in the arch, including wall abnormalities or anatomical varia- tions influencing flow patterns, contribute to the occurrence of dissection in the descending aorta. The majority (54\%) of the patients with TS developed dissection in the ascending aorta, while only $16.4 \%$ developed a dissection in the descending aorta. ${ }^{18}$ Further research is required to determine whether descending aortic dissection can be a result of long-lasting abnormal flow patterns caused by AA abnormalities or increased stiffness as in patients with TS. Consequently, these patients might benefit from more intensive follow-up and earlier preventive surgery.

In addition, our histomorphological results showed abnormalities in the wall of the patients with TS. A hitherto undescribed and striking pattern, which is not scored in the current consensus statement, ${ }^{16}$ was seen in most patients, with compact smooth muscle cell layers and granular 

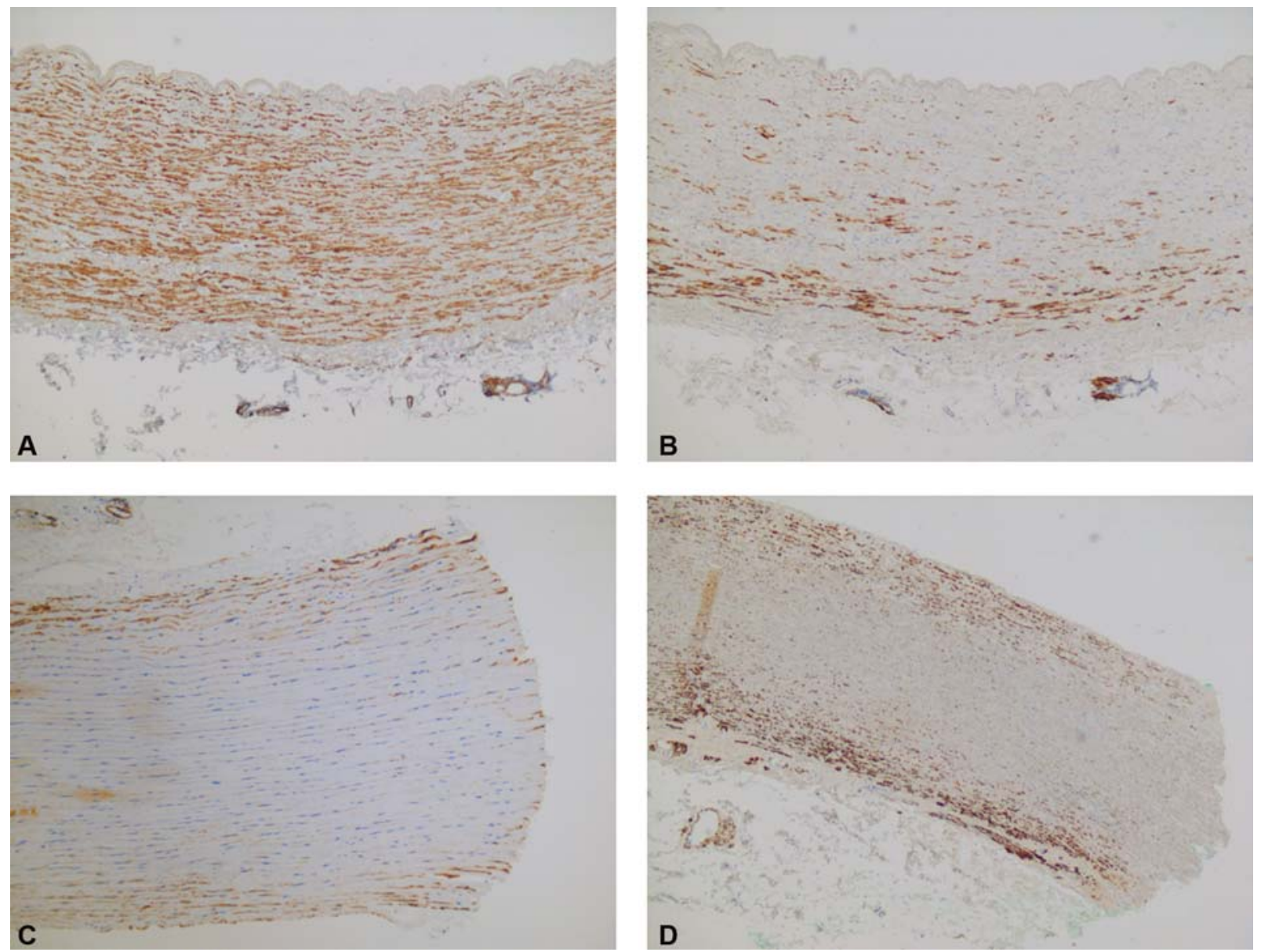

Fig. 5 Immunohistochemical stains of patient with Turner syndrome and healthy control. Top row: (A) immunohistochemical caldesmon and (B) desmin stains of representative aortic explant of controls. Bottom row: (C) immunohistochemical caldesmon and (D) desmin stains of representative aortic explant of Turner syndrome patient, with appreciable loss of staining in the media for both proteins.

deposition of elastin adjacent to lamellae and in the intralamellar tissue. The described compact smooth muscle cell layers and a decrease in the intralamellar space without strong evidence of smooth muscle cell loss indicates a smooth muscle cell volume loss. This finding is supported by contractile fiber attenuation demonstrated by a complete lack of desmin expression, underexpression of caldesmon, and HHF-35, as well as weak expression of PR. Our results are in line with a murine study ${ }^{19}$ that showed decreased smooth muscle cell content and expression of smooth muscle actin in mice with TS. Hinton et al ${ }^{19}$ also found subtle smooth muscle cell misalignment abnormalities that were not present in our patients. The phenomena of decreased smooth muscle cell volume with contractile fiber attenuation and granular deposition of elastin may be key factors in medial destabilization in TS patients. Because we only included patients who had undergone surgery for aortic dilatation, these changes could also be a result of aortic dilatation. However, it is not consistently present in aortic resection specimens from other types of connective tissue disease (such as Marfan's disease or Loeys-Dietz syndrome [own observation and Jain et al. $\left.{ }^{20}\right]$ ). Based on our findings, we suggest that both smooth muscle cell volume and (patterns of) elastin deposition should be included in future grading schemes to evaluate aortic tissue, rather than solely focusing on smooth muscle cell loss and elastin degradation, respectively.

Although histomorphological data confirms that the wall is different in patients with TS, the elasticity measurement of the ascending aorta (ascending aortic distensibility) was not statistically significant different between TS and controls. This might indicate that elasticity measurements of the ascending aorta are impeded by nonoptimal diagnostic power to determine these adverse structural changes within the aortic vessel wall of individual patients with TS. If that is the case, hopefully new imaging developments such as nuclear imaging 21 or computational fluid dynamics ${ }^{22}$ will help to noninvasively visualize the changes in the aortic wall or the abnormal blood flow as a result of abnormal aortic wall behavior. Another reason for our nonsignificantly different ascending aortic distensibility between women with and without TS might be the limited number of participants. Since there seemed to be a tendency to a reduced ascending aortic distensibility in patients with TS, the size of the group might have prevented us from finding a significant difference. Others have also investigated whether the presence of BAV can explain the increased aortic stiffness in patients 
with TS but the results are contradictory. Two studies ${ }^{8,23}$ showed reduced ascending aortic distensibility and increased AA-PWV independently of the presence of BAV, while Devos et $\mathrm{al}^{9}$ showed that only patients with BAV have a reduced ascending aortic distensibility. Our results are more in line with the first study, since we found no effect of BAV on stiffness. The influence of CoA on aortic stiffness in patients with TS has been investigated by Wen et $\mathrm{al}^{10}$ who showed that the aortic distensibility of the descending aorta was only lower among TS patients with CoA. A history of hypertension and specific medical treatment and the effect of the treatment could have caused these ambiguous results in different studies. Our own and former results should be interpreted with caution and verified in a larger cohort of TS patients, taking into account the assumed factors which affect the aortic elasticity.

The aortic stiffness over the entire aorta measured with CFPWV showed no significant difference between patients with TS and healthy controls, which is in line with previous literature. $^{24}$ Important to acknowledge is that CF-PWV uses a conventional measurement of the distance on the body surface. Patients with TS are known to have a fairly abnormal course of the larger vessels with unrolling and tortuosity. ${ }^{9,25}$ Because of these aortic abnormalities, measurement of the aortic length on the outside of the body is likely to fail in patients with TS. We probably underestimated the length of the aorta, therefore, the value of the CF-PWV will be measured lower than it actually is. The classic CF-PWV might not be efficient enough to identify abnormal aortic elasticity with this measurement in patients with TS due to methodological issues.

\section{Conclusion}

Women with TS show a stiffer AA independently of the presence of a BAV or CoA. No differences were found in aortic stiffness between patients with monosomy or mosaicism. In addition, histomorphological changes are found in the ascending aorta. Our histomorphological investigation provided new insights into the structure of the aortic wall in patients with TS. Further research should aim to investigate whether these structural changes are indeed related to the markedly different elastic properties of the ascending aorta in a larger cohort and how we can identify this difference in elastic properties more accurately with the use of new imaging developments.

\section{Funding}

This study was supported by the Dutch Heart Foundation (contract grant number: 2013T093).

\section{Conflict of Interest}

The authors declare no conflict of interest related to this article.

\section{Acknowledgments}

The authors acknowledge the funding support provided by the Dutch Heart Foundation.

\section{References}

1 Stochholm K, Juul S, Juel K, Naeraa RW, Gravholt CH. Prevalence, incidence, diagnostic delay, and mortality in Turner syndrome. J Clin Endocrinol Metab 2006;91(10):3897-3902

2 Matura LA, Ho VB, Rosing DR, Bondy CA. Aortic dilatation and dissection in Turner syndrome. Circulation 2007;116(15):1663-1670

3 Davies RR, Goldstein LJ, Coady MA, et al. Yearly rupture or dissection rates for thoracic aortic aneurysms: simple prediction based on size. Ann Thorac Surg 2002;73(01):17-27, discussion 27-28

4 Gravholt CH, Andersen NH, Conway GS, et al; International Turner Syndrome Consensus Group. Clinical practice guidelines for the care of girls and women with Turner syndrome: proceedings from the 2016 Cincinnati International Turner Syndrome Meeting. Eur J Endocrinol 2017;177(03):G1-G70

5 Carlson M, Airhart N, Lopez L, Silberbach M. Moderate aortic enlargement and bicuspid aortic valve are associated with aortic dissection in Turner syndrome: report of the international turner syndrome aortic dissection registry. Circulation 2012;126(18): 2220-2226

6 Duijnhouwer AL, Bons LR, Timmers HJLM, et al. Aortic dilatation and outcome in women with Turner syndrome. Heart 2019;105 (09):693-700

7 Vlachopoulos C, Aznaouridis K, Stefanadis C. Prediction of cardiovascular events and all-cause mortality with arterial stiffness: a systematic review and meta-analysis. J Am Coll Cardiol 2010;55 (13):1318-1327

8 Schäfer M, Browne LP, Truong U, et al. Aortic stiffness in adolescent Turner and Marfan syndrome patients. Eur J Cardiothorac Surg 2018;54(05):926-932

9 Devos DG, De Groote K, Babin D, et al. Proximal aortic stiffening in Turner patients may be present before dilation can be detected: a segmental functional MRI study. J Cardiovasc Magn Reson 2017; 19(01):27

10 Wen J, Trolle C, Viuff MH, et al. Impaired aortic distensibility and elevated central blood pressure in Turner Syndrome: a cardiovascular magnetic resonance study. J Cardiovasc Magn Reson 2018; 20(01):80

11 Nistri S, Grande-Allen J, Noale M, et al. Aortic elasticity and size in bicuspid aortic valve syndrome. Eur Heart J 2008;29(04):472-479

12 Ong CM, Canter CE, Gutierrez FR, Sekarski DR, Goldring DR. Increased stiffness and persistent narrowing of the aorta after successful repair of coarctation of the aorta: relationship to left ventricular mass and blood pressure at rest and with exercise. Am Heart J 1992;123(06):1594-1600

13 Carlson M, Silberbach M. Dissection of the aorta in Turner syndrome: two cases and review of 85 cases in the literature. J Med Genet 2007;44(12):745-749

14 Bons LR, Duijnhouwer AL, Boccalini S, et al. Intermodality variation of aortic dimensions: How, where and when to measure the ascending aorta. Int J Cardiol 2019;276:230-235

15 Devos DG, Rietzschel E, Heyse C, et al. MR pulse wave velocity increases with age faster in the thoracic aorta than in the abdominal aorta. J Magn Reson Imaging 2015;41(03):765-772

16 Halushka MK, Angelini A, Bartoloni G, et al. Consensus statement on surgical pathology of the aorta from the Society for Cardiovascular Pathology and the Association for European Cardiovascular Pathology: II. Noninflammatory degenerative diseases - nomenclature and diagnostic criteria. Cardiovasc Pathol 2016;25(03): 247-257

17 Mortensen KH, Gopalan D, Nørgaard BL, Andersen NH, Gravholt $\mathrm{CH}$. Multimodality cardiac imaging in Turner syndrome. Cardiol Young 2016;26(05):831-841

18 Wong SC, Cheung M, Zacharin M. Aortic dilatation and dissection in Turner syndrome: what we know, what we are unclear about and what we should do in clinical practice? Int J Adolesc Med Health 2014;26(04):469-488 
19 Hinton RB, Opoka AM, Ojarikre OA, Wilkinson LS, Davies W. Preliminary evidence for aortopathy and an X-linked parent-oforigin effect on aortic valve malformation in a mouse model of Turner syndrome. J Cardiovasc Dev Dis 2015;2(03):190-199

20 Jain D, Dietz HC, Oswald GL, Maleszewski JJ, Halushka MK. Causes and histopathology of ascending aortic disease in children and young adults. Cardiovasc Pathol 2011;20(01):15-25

$21 \mathrm{Kim}$ J, Song HC. Role of PET/CT in the evaluation of aortic disease. Chonnam Med J 2018;54(03):143-152

22 Youssefi P, Sharma R, Figueroa CA, Jahangiri M. Functional assessment of thoracic aortic aneurysms - the future of risk prediction? Br Med Bull 2017;121(01):61-71
23 Pees C, Heno JA, Häusler G, Ertl DA, Gulesserian T, Michel-Behnke I. Aortic elasticity deterioration proves intrinsic abnormality of the ascending aorta in pediatric Turner syndrome unrelated to the aortic valve morphology. Heart Vessels 2018;33(11):1350-1357

24 Ostberg JE, Donald AE, Halcox JP, Storry C, McCarthy C, Conway GS. Vasculopathy in Turner syndrome: arterial dilatation and intimal thickening without endothelial dysfunction. J Clin Endocrinol Metab 2005;90(09):5161-5166

25 Kim HK, Gottliebson W, Hor K, et al. Cardiovascular anomalies in Turner syndrome: spectrum, prevalence, and cardiac MRI findings in a pediatric and young adult population. AJR Am J Roentgenol 2011;196(02):454-460 